\title{
Original Article \\ Phase II study of cisplatin, etoposide and paclitaxel in locally advanced or metastatic adenocarcinoma of gastric/ gastroesophageal junction
}

\author{
Sharma A, Raina V, Lokeshwar N, Deo SVS, Shukla NK, Mohanti BK \\ Department of Medical Oncology, Institute Rotary Cancer Hospital, All India Institute of Medical \\ Sciences, New Delhi, India
}

Correspondence to: Atul Sharma, E-mail: atul1@hotmail.com

\section{Abstract}

BACKGROUND: Unresectable and metastatic gastric cancers carry a poor and dismal prognosis. Several phase II studies have identified effective anticancer drugs. AIMS: To evaluate safety and efficacy of low-dose cisplatin, etoposide and paclitaxel (CEP) based combination chemotherapy in locally advanced or metastatic adenocarcinoma of gastric/gastroesophageal junction. SETTING AND DESIGN: Prospective single-arm phase II study. MATERIALS AND METHODS: Thirty-three patients were enrolled onto this study, out of which, all but one received cisplatin $15 \mathrm{mg} / \mathrm{m}^{2}$, etoposide $40 \mathrm{mg} / \mathrm{m}^{2}$ and paclitaxel $50 \mathrm{mg} / \mathrm{m}^{2}$, given on day 1 and 4 every week for three weeks in a 28-day cycle. Survival analysis was done using SPSS program. RESULTS: Median age of group was 56 years. Twenty-five were males. Twenty-nine had metastatic/inoperable disease and four patients had recurrent disease. Liver was the commonest metastatic site seen in 15 patients. With a median of 2 cycles per patient, a total of 76 cycles was administered. Grade III or IV toxicity were seen in 11 (35\%) patients; diarrhea, 5 patients; vomiting, 3 patients; and neutropenia, 7 patients, 5 of whom also had fever). One patient died of neutropenic fever. Best responses, seen in 32 evaluable patients, were 2 CR (6.1\%), 21 PR (63\%) and 3 SD (9.2\%). Four patients were considered operable after chemotherapy. With median follow-up of 11 months in surviving patients, median OS was 10 months and PFS was 8 months. Median OS was 13 months in responders versus 8 months in nonresponders $(P=0.04)$. Seven patients survived $>12$ months. CONCLUSION: Combination of low-dose CEP shows good clinical response and an acceptable toxicity profile in advanced or metastatic adenocarcinoma of gastric/gastroesophageal cancers. Whether addition of $5 \mathrm{FU}$ or capecitabine adds to the benefit should be explored. This may be tested with other standard/conventional protocols in a randomized fashion.

Key Words: Cisplatin, etoposide, gastric, gastroesophageal, paclitaxel.

Carcinoma of stomach is one of the leading causes of cancer-related mortality. Screening has helped in bringing down gastric cancer-related mortality by early detection and aggressive surgery in Japan, but elsewhere, about two-thirds of patients present in advanced unresectable stage, where cure is unlikely. These patients are managed with palliative chemotherapy, radiotherapy and/or surgery. Median survival of these patients ranges from 5-7 months. Adenocarcinomas of lower one-third of esophagus and gastroesophageal junction behave like that of gastric cancer.

Chemotherapy with or without radiotherapy, though used in adjuvant setting, is also used in advanced/ metastatic cancers. ${ }^{[1]}$ The most commonly used agents 
are 5 fluorouracil, mitomycin, doxorubicin, etoposide, cisplatin and taxanes either alone or in various combinations. Response rates vary from 21 to $53 \%$ with different combinations and permutations. Various single-arm studies have shown median survival of 3.5 to 11 months. ${ }^{[2-4]}$ Reported response rate with combination of DDP and VP-16 is $18 \% \cdot{ }^{[5]}$ In randomized trials, DDP, 5 FU and PELF (cisplatin, epirubicin, 5 FU, folinic acid) have been demonstrated to be more effective than FAM. ${ }^{[6-8]}$ Despite high responses, median survival has been below 10 months. Paclitaxel is an effective agent in esophageal and GE junction tumors. ${ }^{[9]}$ Based upon activity of DDP (P), VP-16 (E) and paclitaxel $(\mathrm{T})$, a combination of these drugs (TPE/CEP) was developed and tried by Lokich $e t$ al ${ }^{[10]}$ with almost $100 \%$ responses in locally advanced esophageal and GE junction tumors. Based on encouraging results from this phase II study, we decided to use this combination in unresectable/metastatic adenocarcinoma of GE junction and stomach at our center; and with this, we also intended to evaluate response rates and survival.

\section{Materials and Methods}

This was a prospective single-arm phase II study. Study period was from May 2000 to February 2004.

\section{Inclusion and exclusion criteria}

To be eligible for this study, patients had to have advanced unresectable or metastatic adenocarcinoma of stomach or gastroesophageal junction, measurable disease, no prior radiotherapy; adjuvant prior chemotherapy, if given, should have been completed 6 months ago. The patients should have ECOG performance status of $<3$; and adequate liver, kidney and bone marrow functions. All patients signed informed consent before enrollment onto study.

\section{Treatment regimen}

CEP regimen consisted of Cisplatin $15 \mathrm{mg} / \mathrm{m}^{2}$, Etoposide $40 \mathrm{mg} / \mathrm{m}^{2}$, Paclitaxel $50 \mathrm{mg} / \mathrm{m}^{2}$, IV infusion (with antiemetic) given on day 1 and 4 for 3 consecutive weeks followed by 1 -week rest. Cycles were repeated every 28 days. If neutrophil count was below $1000 / \mathrm{cmm}$ and/or platelet count below $75,000 / \mathrm{cmm}$, chemotherapy was withheld until recovery of counts. Prophylaxis growth factors were not used.

\section{Response and toxicity criteria}

Response assessment was done after every 2 cycles with upper GI endoscopy and CT scan. Subjective assessment was done after each cycle. Complete response (CR) was defined as disappearance of all evidence of disease lasting for 4 weeks. Partial response $(\mathrm{PR})$ required more than $50 \%$ regression in the product of the perpendicular diameters of indicator lesions for a minimum of 4 weeks without any new lesions. Progressive disease was defined as appearance of any new lesion or increase by $25 \%$ or more in size of indicator lesion. Stable disease (SD) was the one that did not met any of above criteria. National Cancer Institute Common Toxicity Criteria (NCI-CTC) were used to assess drug-induced toxicity. After 2 or 3 cycles, eligible patients were considered for further treatment with surgery and or radiotherapy.

\section{Statistical analysis}

Survival analysis was done on intention to treat basis. Overall survival and time to progression were calculated from the time of initiation of treatment. Statistical analysis and Kaplan and Meier survival curves were plotted using SPSS program.

\section{Results}

Demographic features of 33 patients enrolled onto this study are shown in [Table 1]. Median age was 56 years (range 32-70 years). Twenty-five were males. All patients had 'pain' as one of the presenting symptoms; along with this, 8 patients $(25 \%)$ had gastric outlet

\begin{tabular}{|c|c|}
\hline Characteristic & Number of patients \\
\hline Total number & 33 \\
\hline Treatment received & 32 \\
\hline Median age in years (range) & $56(32-70)$ \\
\hline Sex- males & 25 \\
\hline \multicolumn{2}{|l|}{ Site of tumor } \\
\hline Gastric & 29 \\
\hline GE junction & 04 \\
\hline Recurrent disease & 04 \\
\hline First presentation & 29 \\
\hline \multicolumn{2}{|l|}{ Site of metastates } \\
\hline Liver & 15 \\
\hline Ascites / peritoneum & 12 \\
\hline Grade III/IV toxicity & 11 \\
\hline CR & 02 \\
\hline PR & 21 \\
\hline SD & 03 \\
\hline OR & $23(69 \%)$ \\
\hline
\end{tabular}

$\mathrm{CR}=$ complete response, $\mathrm{PR}=$ partial response, $\mathrm{SD}=$ stable disease, $\mathrm{OR}=$ overall response 
obstruction. ECOG performance status was IV in 4 patients, III in 25 patients and II in 4 patients. Twentynine $(90 \%)$ patients had metastatic or inoperable stage at the time of presentation, whereas there was recurrence in 4 patients after initial surgery. Twenty-two patients had more than one metastatic site. Commonest metastatic sites, as expected, were liver and ascites, seen in $15(46 \%)$ and $12(40 \%)$ patients respectively. Even though patients were planned for 3 or 4 cycles, a total of 76 cycles only could be delivered with a median of 2 cycles (range 1-4). Many patients could not continue chemotherapy beyond 2 cycles because of economic reasons rather than toxicity or ineffectiveness.

\section{Response evaluation}

Thirty-two out of 33 patients were evaluable for response. One patient dropped out of study before treatment was commenced. Symptomatic improvement was seen in all the patients at the end of first cycle itself, resulting in significant decrease in pain and control of gastric outlet obstruction. Best responses seen were 2 CR (6.1\%), $21 \mathrm{PR}(63 \%)$ and 3 SD (9.2\%). Six patients progressed while on therapy. All these responses were documented by endoscopy and CT scan. Four patients were considered suitable for surgery at the end of three cycles of chemotherapy. However, after exploration, tumor of only one patient could be resected. A patient who had nodal relapse after initial surgery was given radical radiotherapy to lymph nodal area after complete response to chemotherapy.

\section{Survival}

After median follow-up of 11 months (range 1-18 months), median OS was 10 months and median PFS was 8 months [Figures 1]. Median OS was 13 months in patients who had response to chemotherapy versus 8 months in nonresponders $(P=0.04)$. Seven patients $(22 \%)$ survived $>12$ months. Twenty-six patients died -

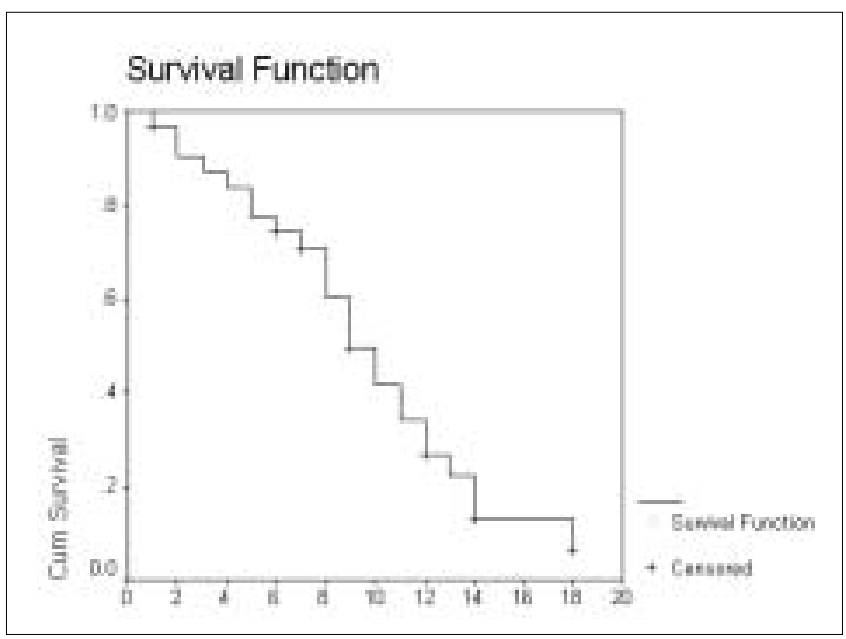

Figure 1: OS in months
25 because of disease progression and 1 after chemotoxicity. Of the remaining 7 patients, 2 are alive without disease and 3 are alive with disease. Follow-up information about 2 patients is not available at the time of analysis. (This includes one patient who was not treated.)

\section{Toxicity}

Combination of CEP was generally well tolerated with manageable toxicities. Eleven (35\%) patients had single or multiple grade III or IV toxicity requiring dose modifications; diarrhea, 5 patients; vomiting, 3 patients; neutropenia, 7 patients, 5 of whom also had fever). One patient died of neutropenic fever and pneumonia. Grade III and IV toxicities seen in the study are listed in Table 2.

\section{Discussion}

Advanced and unresectable gastric or GE junction cancers carry poor prognosis. Many chemotherapeutic agents have been shown to have antitumor activity; however, median survival does not exceed 5-7 months in most of the reports. Cisplatin, paclitaxel and etoposide possess antitumor activity in these tumors. Many newer agents like irinotecan, capecitabine, docetaxel and oxaliplatin also possess anticancer activity in gastric cancer. The combination of CEP used in this phase II trial is similar to the one used by Lokich. ${ }^{[10]}$

5 FU and anthracyclins have been other agents commonly used in these settings. Popular regimen of FAM has been compared with FAMTX with response rates of 9 and $41 \%$ respectively. ${ }^{[7]}$ In another trial, ECF (epirubicin, cispaltin and 5FU) has demonstrated superior response rates as compared to FAMTX ( $5 \mathrm{FU}$, methotrexate and doxorubicin). The response rates were 45 and $21 \%$ respectively, which is equivalent to this phase II study. ${ }^{[1]}$ Even though the number of patients is small in the current series, it is noteworthy that all

Table 2: Grade III and IV toxicity encountered

\begin{tabular}{|c|c|}
\hline Toxicity & Number of patients* \\
\hline Diarrhoea & 06 \\
\hline Vomiting & 03 \\
\hline Neutropenia & 10 \\
\hline Neutropenic fever & 05 \\
\hline Thrombocytopenia & 03 \\
\hline Toxic death & 01 \\
\hline No significant toxicity & 21 \\
\hline
\end{tabular}


the patients who received the therapy experienced symptomatic improvement after first cycle itself. This multifractionated protocol is aggressive in the sense that close to $20 \%$ patients had grade III or IV neutropenia. This hematological toxicity was manageable and only one required growth factor; this is despite the fact that about $90 \%$ patients had ECOG performance status of III or IV. In this trial, we could not duplicate the response rates of more than $90 \%$, as reported in the previous study. ${ }^{[10]}$ One possible reason is that median number of cycles in our study was 2 as compared to 4 in the original study by Lokich et al. Another notable difference in our study and the previous study ${ }^{[10]}$ was that only 5 of our patients could be considered for further therapy (surgery or RT) as compared to 19 out of 25 patients in the study by Lokich. Again, the explanation may be, because of less number of cycles, there were not adequate responses. However, it is to be remembered that as compared to locally advanced patients in earlier series, $90 \%$ of our patients had metastatic disease, which was a clearly inoperable situation. The median survival of 10 months in our series is largely attributable to chemotherapy alone. Survival of 12.5 months was reported by Lokich et al, but it is to be remembered that about $80 \%$ of patients in their series also received local consolidation, which must have contributed to survival. We have demonstrated that patients whose disease has responded to chemotherapy had median survival of 13 months as compared to 8 months in nonresponders $(P=0.04)$. We hypothesize that if we can increase these responses by addition of another agent like $5 \mathrm{FU}$, which is known to have significant activity in gastric cancers or capecitabine, we should be able to increase the survival. In one of the recent phase III studies, DCF (docetaxel, cispaltin and $5 \mathrm{FU}$ ) has been compared with cisplatin and $5 \mathrm{FU}$. The response rates $(39 \%$ vs $23 \%)$ and overall survival (10.2 months vs $8.5 \%$ ) were significantly higher in DCF arm, thereby confirming the role of taxane. ${ }^{[12]}$ There is a need to test this combination of CPE after adding oral capecitabine or 5 FU to other standard protocols of EAP (cisplatin, doxorubicin, etoposide), ECF or one of the 5FUcontaining combinations. It is possible that with the use of new combinations, responses as well as survival may be better; in other words, what we see today for advanced colorectal cancer may be true for gastric cancers tomorrow.

\section{Conclusion}

Poor survival of advanced unresectable cancer of
stomach/GE junction has led to initiation of various clinical trials using different chemotherapeutic drugs in different combinations. This 3-drug combination of CEP is safe, effective and well tolerated. It is possible that by increasing responses, survival will also increase. This combination warrants further studies in combination with $5 \mathrm{FU}$ or capecitabine in randomized fashion.

\section{References}

1. Kohne $\mathrm{CH}$, Wils JA, Wilke HJ. Development in the treatment of gastric cancer in Europe. Oncology (Huntingt) 2000;14;22-5.

2. Cocconi G, DeLisi V, DiBlaso B. randomized comparison of $5 \mathrm{FU}$ alone or combined with mitomycin and cytarabine (MFC) in the treatment of advanced gastric cancer. Cancer Treat Rep 1982;66:1263.

3. Cullinan SA, Moertel CG, Fleming TR, Rubin JR, Crook JE, Everson $\mathrm{LK}$, et al. A comparison of three chemotherapeutic regimens in the treatment of advanced pancreatic and gastric carcinoma. JAMA 1985;253:2061-7.

4. Gastrointestinal Tumor Study Group. Trianzinate and platinum efficacy in combination with 5-fluorouracil and doxorubicin:results of a three-arm randomized trial in metastatic gastric cancer. J Natl Cancer Inst 1988;80:1011.

5. Elliot TE, Moertel CG, Wieand HS, Hahn RG, Gerstner JB, Tschetter LK, et al. A phase II study of the combination of cispaltin and etoposide in the therapy of advanced gastric cancer. Cancer 1990;65; 1491.

6. Kim NK, Park YS, Heo DS, Suh C, Kim SY, Park KC, et al. a phase III randomized study of 5 fluorouracil and cisplatin versus 5 fluorouracil, doxorubicin and mitomycin $C$ versus 5 fluouracil alone in the treatment of advanced gastric cancer. Cancer 1993;71:3813-8.

7. Wils J, Klein HO, Wagener DJ, Bleiberg $\mathrm{H}$, Reis $\mathrm{H}$, Korsten $\mathrm{F}$, et al. Sequential high dose methotrexate and fluorouracil combined with doxorubicin: A step ahead in the treatment of advanced gastric cancer- A trial of the European Organization for Research and Treatment of Cancer Gastrointestinal Tract Cooperative Group. J Clin Oncol 1991;9:827-31.

8. Cocconi G, Bella M, Zironi S, Algeri R, DiCostanzo F, DeLisi V, et al. Fluorouracil, doxorubicin and mitomycin combination versus PELF chemotherapy in advanced gastric cancer: A prospective randomized trial of the Italian oncology group for clinical research. J Clin Oncol 1994;12:2687.

9. Ajani JA, Fairweather J, Dumas P, Patt YZ, Pazdur R, Mansfield $\mathrm{PF}$, et al. phase li study of Taxol in patients with advanced gastric cancer. Cancer J Sci Am 1998;4:269-74.

10. Lokich JJ, Sonneborn IT, Anderson NR, Bern MM, Coco EV, Dow E, et al. combined Paclitaxel, Cisplatin and Etoposide for patients with previously untreated esophageal and gastroesophageal carcinomas. Cancer 1999;85:2347-51.

11. Cocconi G, Carlini P, Gamboni A, Gasperoni S, Rodino C, Zironi S, et al. Cisplatin, epirubicin, leucoverine and 5-fluourouracil (PELF) is more active than 5- fluourouracil, doxorubicin and methotrexate (FAMTX) in advanced gastric carcinoma. Ann Oncol 2003; 14:125863.

12. Ajani JA, Van Cutsem E, Moiseyenko V, Tjulandin S, Fodor M, Majlis $A$, et al. Docetaxel (D), cisplatin, 5 fluorouracil compare to cisplatin (C) and 5 fluorouracil (F) for chemotherapy naïve patients with metastatic or locally recurrent, unresectable gastric cancer (MGC):Interim results of a randomized phase III trial (V 325). Proc Am Soc Clin Oncol 2003;22:249(abstr 999). 